tion. He mentions also the bromides of mercury, and gives the dose of the subbromide (bromide) from one to two grains, and that of the perbromide (bibromide) frop one-sixteenth to one-fourth of a grain; but I have usually found that the subbromide of mercury exerts a greater power than its corresponding salt, calomel.

Having been in the habit of prescribing the bromides of mercury during the past five years, and being impressed with their value as therapeutical agents, I wish concisely to point out their general effects, in the hope of enlisting others in this investigation; so that, by long and continued observation, we may together accumulate such knowledge of their powers as may enable us more accurately to judge of their merits, as compared with those of the chlorides.

I have found, with the protobromide or subbromide, that a less dose than of calomel is required; that it acts more agreeably and effectually, both as a cholagogue and purgative, without the depressing results so often observed to follow the exhibition of calomel. It seems to be useful in promoting the absorption of hypertrophied glandular and other morbid tissue with less constitutional disturbance than the chlorides. I have administered it, with apparent great relief, in cases of biliary congestion of the liver, in chronic hepatitis with enlargement, and in those skin-diseases which yield best to the influence of mercury. I might quote some interesting cases of large anomalous tumours of the abdomen sensibly diminishing, and in two instances wholly disappearing, under the use of these remedies-viz, the protobromide of mercury in half-grain doses, and the perbromide in the form of an ointment of four grains to the ounce of cold cream; also of uterine tumefactions and morbid deposits around the cervix uteri and in the connective tissue of the pelvis, treated with pessaries of the perbromide, as well as the ointment externally. I could cite other instances of acute and chronic inflammatory disease in which the bromides have been apparently of great use; but my observations have been as yet so limited, and the difficulties of making perfectly correct observations on the action of a new remedy are so great, that $I$ think it best to forego such means of proving its value till a mass of evidence can be adduced which may better test the accuracy of my conclusions. With your permission, therefore, sir, I would here ask those members of the Association who $\mathrm{man}^{k}$ try these remedies to note their effects, and kindly to favour me vith a report of their observations, so that I may, at a future time, publigh the result of our united efforts; since to arrive at any definite ideas of the action of the bromides of mercury will, I feel sure, require the careful work of many observers.

This short paper, then, claims to be put forward, not as a complete account of a new remedy, but as a notice to the profession, with the view of stimulating others to take up and investigate the subject.

\section{ON CARIES OF BONE.}

BY HOLMES COOTE, Esq., Surgeon to St. Bartholomew's Hospital, London.

I HAVE been long convinced that the results of operations undertaken for the removal of carious bone are unsatisfactory. We see case after case in which the surgeon gouges away as much as he dare of the dis. eased structure; and yet he finds, when the period for the cicatrisation of the wound comes about, that the fistulous sinuses still remain open, and that the annoyances to the patient are as great as before. A second and a third operation may be recommended and performed, and still the relief is incomplete, until, finally, a cure is effected by natural processes.

These reflections lead naturally to the question, What is caries? Is the disease of such a nature as to admit of beneficial treatment by surgical operation?

We find, in reference to this question, no small difference of opinion among surgical authorities. Some mean by the term, simple ulceration of bone; others, chronic suppuration of the cancellous tissue. Mr. Stanley often defined it, in my presence, as "unhealthy ulceration of bone." Mr. Erichsen speaks of it as a disease characterised by increased vascularity, softening, and ultimate disintegration of the osseous tissie.

Perhaps the last definition expresses best the true nature of caries; but it is wanting in accuracy of detail.

Caries may be said to have its seat, with scarce exception, in the can. cellous structure of bone, such as the bodies of the vertebra, the bones of the carpus and tarsus, or the spongy extremities of the long bones. As already observed, the disease commences with increased vascularity (ostitis or osteomyelitis); and I think that I have never known it to be transmitted from the soft parts to the bone. It has always commenced in the bone itself. As the disease proceeds, it causes molecular disintegration of the osseous tissue, which comes away in the form of minute fragments mixed with the pus. After a time, larger portions may perish and become detached; but, unlike that which is observed in simple necrosis, these carious sequestra do not represent the whole of the diseased part. One of the most intractable forms of caries is that termed caries fungosa (Volkmann), which is characterised by both ulcerative destruction and the production of an abundant granulation. The bones become mouldering, osteoporotic and thinned, in parts absolutely destroyed, the medullary space being filled by a reddish-coloured marrow. The granulations lose their florid red colour and become pale; there is an abun. dant deposit of fatty matter in the neighbourhood; and the discharge becomes offensive and discoloured. It is this form of caries which especially attacks the bones of the carpus and tarsus. Portions of eroded bone may be detached, but the morbid action extends far beyond their sphere, and usually more bones than one are involved in different stages of the same disease. The pressure of the granulations is followed by absorption and alteration in contour, while the internal disintegration may terminate in leaving little more than an external shell of compact, but thinned and light tissue.

This is the disease, and such the conditions of the morbid parts, which we in modern surgery attempt to rectify by the operation of gouging away the diseased bone. The results have not realised my ex. pectations, except in cases where bone, already detached, admitted of easy removal, or where an emptied cloaca, surrounded by healthy bone, could be scraped so as to relieve it of its soft and unhealthy lining.

Under the most favourable conditions, the prognosis is far from good; but I am inclined to doubt whether the patient's condition would not be better after a period of twelve months, and simply kept at rest and in good air, than if he had undergone the usual operation.

\section{A CONTRIBUTION TO BABY-FARMING. \\ BY HERMANN BEIGEL, M.D., M.R.C.P., Physician to the Metropolitan Free Hospital.}

THe following interesting case illustrates some of the evils of the system of baby-farming, which have been so ably exposed in the reports which recently appeared in the columns of the BRITISH MEDICAL JOURNAI.

Rebecca Levy, now twenty-four years of age, was, soon after birth, possessed of a loud and clear voice. When she was one month old, her mother fell ill, and was obliged to become an in-patient at a hospital, and to give her baby in charge of a woman to be nursed. Very little care was, however, taken of the child, who was left by her nurse in a room with the door closed, with very little food, and who consequently cried nearly the whole day. This continued for nearly twelve months, when the mother returned home, and took the child back, the latter being but ill-fed and perfectly hoarse. The girl never recovered her voice, and only experienced a difference in the degree of hoarseness. During the last few months, this condition had become at times alarming; fits of suffocation sometimes setting in, interrupting sleep, which was always ac. companied by a whistling, rattling noise. The patient could never lie on her back without experiencing one of these suffocating attacks; and was, therefore, obliged to remain in a sitting posture during the night. On the 27th of May last, she came to the Metropolitan Free Hospital in a state of suffocation, and was admitted as an in-patient under my care. Her breathing was at this time extremely laborious; the ordinary muscles of respiration, as well as the auxiliary muscles, were in power. ful action; the alæ of the nose were working violently; the visible mu.

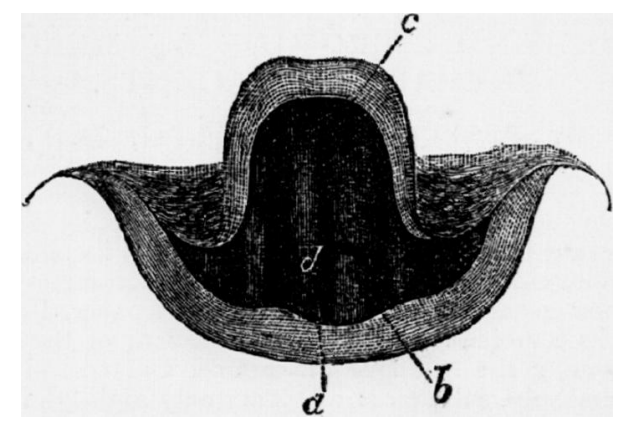

cous membranes were livid; perspiration covered her face and forehead; her speech was very hoarse and thick; and, from want of breath, she could only pronounce short interrupted sentences.

Laryngoscopic Examination. - The pharynx was red. The larynx and vocal cords were as illustrated in the accompanying woodcut. Both 
vocal cords had entirely lost their white appearance, and were converted into flesh-like masses. They were, moreover, detached from their insertion into the arytenoid cartilages; and the left vocal cord $(c)$ was ruptured through its whole breadth at about its lower third $(b)$. There was no movement whatever of the vocal cords visible, except the lower portion of the left cord, which acted as a kind of valve, floating inwardly at each inspiration, and outwardly at each expiration; the right vocal cord and the upper portion of the left hanging down perpendicularly, and lying closely applied to one another, leaving no rima whatever. The false vocal cords were much swollen, and partly covered the former. It must be remarked, that the lower portion of the left vocal cord did not present the fleshy appearance described above, but had partly retained its white appearance.

The symptoms were, in fact, so urgent, that I contemplated perform. ing tracheotomy; but, owing to the excellent action of the lower portion of the left vocal cord, as described above, I determined to try the effect of the local application of a strong solution of nitrate of silver; and afterwards directed her to suck ice, and to apply it externally to the larynx. After twenty-four hours, the inflammation had decreased so far under this treatment, that the patient was able to lie on her back, and to respire with comparative ease. After applying the nitrate of silver four times, the vocal cords had returned to that state which, in this patient, must be considered normal. The cords, being now considerably diminished in size, became moveable, and presented, at each respiration, a peculiar appearance. The right vocal cord, as well as the two parts of the left one, instead of performing the movements seen in a healthy larynx, were floating altemately inwards and outwards at each movement of inspiration, in the same manner as was the case with the lower portion of the left vocal cord during the height of inflammation.

The patient remained in the hospital up till June 17th, without experiencing any further inconvenience, except that her voice continued thick and hoarse-a state which does not admit of any remedy. She was discharged from the hospital, after improving considerably in her general health.

There can be no doubt as to the etiology of this case. The continued overstraining of the vocal cords by constant screaming was, at such an early period of life, sufficient to detach both of them from their insertion, and to tear the left vocal cord asunder; and is, therefore, distinctly traceable to the neglect of the child arising from the system of baby-farming. I am not aware that any similar case has been placed upon record.

\section{THERAPEUTICAL MEMORANDA.}

[Under this head, we shall publish from time to time, as materials accumulate, short records of remarkable cases in practice which are suffi. ciently rare, interesting, or instructive, to deserve record, but do not call for lengthened statement or comment. Brevity and point should be the valuable characteristics of cases forwarded for this column.]

\section{CASE OF EPILEPTIC CONVULSIONS TREATED BY GALVANISM.}

- By Fred. Wm. Parsons, L.R.C.P.Lond, etc., Islip, Oxon.

MRS. B., aged 36, a strong healthy-looking woman, had six children previously, all good times; the last one eight years ago. There is history of insanity. Her mother is in an asylum, and two of her sisters are of unsound mind, and the woman herself is of very weak intellect. She last menstruated on February 27th, 1866, and first felt the movements of the child the first week in July. About October 24th, she had a sanguineous discharge, with pains; the discharge was rather profuse; but upon lying down, it went off, and she felt the movements of the child up to November 2oth. On the evening of November 26th, pains came on regularly; but at three o'clock the next morning she had a fit, and I was sent for. I saw her about eight o'clock, and she was then in a fit, lying on her left side, mouth drawn to the left, and bloody froth issuing from it; prosthotonos; tonic spasm; skin quite cool and dry; pulse 84 ; eyes turned up; pupils normal. The fit lasted about four minutes, when she became partly sensible but very sleepy, and in about ten minutes had another fit. She continued in this way for about an hour, the fits be. coming more frequent and severe, and the patient quite comatose in the intervals. There were now no pains; through the abdominal walls the uterus felt quite flaccid; the os uteri was dilated about the size of a shil. ling, and was dilatable. The presentation was made out to be normal. After the first fit, I gave a good dose of calomel and jalap, but it was all returned during the next fit. There being no uterine contraction what- ever, I determined to try the effect of galvanism externally to the uterns, which had the desired effect immediately, the beg of membranes becom. ing tense, and protruding through the os. The membranes were then ruptured, and the head quickly descended. Short forceps were applied, and the child was delivered in three-quarters of an hour from the commencement of the galvanism. The after-birth followed in about fifteen minutes, and there was very little hremorrhage after, the uterus con. tracting regularly and firmly. Galvanism was continued some few min. utes after the placenta had been expelled, to ensure perfect contraction. The child was born dead, and appeared to have been so several days. The patient remained perfectly insensible all day, and at 9 P.M. I ordered a mustard-plaster to the back of the neck and half way down the spine; towards the next morning she became partly sensible, and passed about a pint of urine, which was thrown away before I could see it. The next day (November 29th) at I I A.M, she had passed no water for twenty-eight hours. About two pints of very fotid urine were drawn off, the colour of dark sherry, becoming semi-solid on heating; the catheter was blackened during the operation. There was considerable abdominal tenderness, which was relieved in a day or two by hot fomentations and a mixture of ether and morphia. From this time she con. tinued to improve. The urine was drawn off regularly for about a weele, and gradually became less albuminous and disagreeable. During her recovery she had several attacks of pain in the bowels, but it was almost immediately relieved by the ether and morphia. When I saw ber a week ago, she said she felt quite well, except rather weak. There are two points I think well worthy of notice in the above case. 1 . Immediately the galvanism was commenced the fits ceased, and they have never since returned. 2. The speedy action of the uterus and the ease with which delivery was accomplished, and the very good recovery the woman has made.

\section{TREATMENT OF DIABETES BY PEROXIDE OF HYDROGEN.}

By Samuel J. Bayfield, Esq.

A Gentleman, aged 46 , engaged in commerce, of steady habits, and a moderate liver, suffered from diabetes, for a year and a-half, and had been the whole time under treatment of some of the best practitioners in London, and receiving no benefit, lost both faith and patience, and applied to me; after hearing his statement, and examining his prescriptions, I felt I could not give even feeble hopes of benefit. But it occurring to me that Dr. Day of Geelong, some time since, published in the Lancet the result of the successful treatment of a case under unfarourable circumstances by the use of the ethereal essence of the peroxide of hydrogen in drachm doses three times a day in distilled water, I was determined to try the effect of the remedy upon my patient. I began with half-drachm doses, and gradually increased to the drachm. I also materially relaxed the rigidness of his diet, and discontinued much that was disagreeable and objectionable for one more consonant to his taste and appetite. I have much pleasure in announcing, after about ten weeks steady perseverance, with occasional fluctuations, he quite recovered, and has kept well for several weeks; he is now in the country for the benefit of change of air; he is gaining both strength and flesh ; and he writes home in a strain both hopeful and thankful. I have not troubled you with daily details, for they would be uninteresting and tedious.

\section{OBSTETRIC MEMORANDA.}

[UNDER this head, we shall, from time to time, as materials come to hand from correspondents, publish records of cases remarkable in themselves, or illustrating points of interest in obstetric practice, therapeutic or manipulative. We shall probably in this way preserve from oblivion the notes of very many useful and instructive occurrences in private practice; for the great obstetric experience is that-for the most part hitherto unwritten - of the great body of general practitioners throughout Great Britain. We will only ask those who may forward cases for record, to relate them with the utmost brevity, and equally to condense any appended remarks.]

\section{PLACENTA PRAVIA WITH TWINS BOTH PRESENTING ABNORMALLY.}

By IIUGH Norris, Esq., South Petherton.

M RS. S., aged 3I, pregnant for the fifth time, had had sudden profuse flooding between the sixth and seventh months. She sent for me on $M$ arch 14th, 186\%. She was last confined in June 1863 , when, from 